\title{
Structure and Toxicity of the Prefibrillar Aggregation States of Beta Peptides in Alzheimer's Disease
}

\author{
Angelo Perico, Denise Galante and Cristina D’Arrigo \\ Institute for Macromolecular Studies (ISMAC, CNR)
}

Italy

\section{Introduction}

As in several neurodegenerative diseases, Alzheimer's disease (AD) arises when proteins fail to get their functional fold and aggregate in amyloid fibrils (Chiti \& Dobson, 2006; Chiti et al., 2002). The Abeta peptides (A $\beta)$, proteolytic products generated from beta-amyloid precursor protein (APP), are the subunits of amyloid fibrils that deposit in brain (Forloni et al., 2002; Selkoe, 1996, 2004). A $\beta$ aggregates consist of a mixture of A $\beta$ peptides around 40 aminoacids having different N- and C- termini (Russo et al., 2000). It is now largely accepted that the pathogenic species in $\mathrm{AD}$ are the toxic oligomeric aggregates of $\mathrm{A} \beta$ that assembly in the brain before the formation of amyloid fibrils (Chiti \& Dobson, 2006; Kirkitadze et al., 2002; Walsh \& Selkoe, 2007; Yankner \& Lu, 2009). Oligomers extracted from AD brain potently impair synapse structure and function (Shankar et al, 2008). The structural characterization and neurotoxicity of these oligomeric aggregation states, even though very difficult to achieve due to the fast aggregation processes involved, to the heterogeneous peptide contents and transient organization, is of primary importance in the study of $\mathrm{AD}$ pathogenesis (Jahn et al., 2010).

Monomeric $A \beta$ may assume in water a $\beta$-hairpin conformation stabilized by intramolecular $\mathrm{H}$-bonds and antiparallel $\beta$-strands that lead to intermediate oligomeric states of $A \beta$ subunits with intermolecular H-bonds and parallel $\beta$-strand organization (Campioni et al., 2010; Ono et al., 2009; Roychaudhuri et al., 2009; Sandbergh et al., 2010). More evidence of the structure of oligomers and protofibrils is required that may be obtained by TEM and AFM (Apetri et al., 2006; Pastor et al., 2008; Yoshiike et al., 2007). Recently, new AFM techniques enabled to image a dynamic of $A \beta$ oligomer formation, consistent with the hairpin conformation hypothesis (Mastrangelo et al., 2006). Changing the peptide composition of these prefibrillar aggregates, significant differences in packing strength and solvent exposure are eventually achieved that may be strictly related to cell toxicity.

Between the different species of water-soluble $\beta$-peptides, found in abundance in the fibril brain deposits (full length $A \beta 1-42, A \beta 1-40$ and N-terminal truncated $A \beta s$ ), $A \beta p y 3-42$, a peptide N-terminal truncated at residue $3(\mathrm{Glu})$ and further modified by cyclization of Glu to pyroglutamic acid pyGlu, (catalysed, both in vitro and in vivo, by glutamynil cyclase enzyme, upregulated in the cortices of AD individuals (Shilling et al., 2008)), may have an important role in the cooperative process leading to $\mathrm{AD}$. The $\mathrm{N}$ termination at residue 3 in 
A $\beta p y 3-42$ may change the attitude of the full length A $\beta 1-42$ to aggregate gradually to a fibril organization, heading the $\beta$-peptide to a fast aggregation towards a relatively stable and toxic soluble oligomeric state.

Recently, the faster aggregation kinetics after incubation time of the N-terminal and pyromodified peptides relative to the full length $A \beta$, were demonstrated together with significant morthological differences in the pure or mixed aggregation states ( $\mathrm{D}^{\prime}$ Arrigo et al., 2009; Shilling et al., 2006). Demuth and coll. gave indications that prevention of formation of pyroglutamate-modified N-terminally truncated A $\beta$ s, such as A $\beta p y 3-42$, by inhibition of glutaminyl cyclase, may represent a new therapeutic strategy for alleviating amyloidoses (Shilling et al., 2008). In general, N-terminal pyroglutamyl modifications increase the aggregation propensity and also enhances resistance to degradation by proteases (Shilling et al., 2004, 2006). Prevention of formation of pyroglutamate-modified N-terminally truncated $A \beta s$ may be beneficial for AD patients. The ratio of soluble $A \beta$ species may dictate the role of these aggregates on the AD amyloidosis (He \& Barrow, 1999): particularly the toxic effect of water-soluble $A \beta$ on neurons seems to be related to the predominance of Apy3-42 (Russo et al., 2002). This finding reinforces the idea that an adequate characterization of the oligomeric and prefibrillar states in terms of peptide content, structure and toxicity may be a promising appropriate strategy to design innovative therapies.

The intermedium water-soluble $A \beta$ aggregation states and their assembly process, with a particular attention to the role of $A \beta p y 3-42$, will be the focus of the chapter.

In section 2., a review of the efforts to characterize the structure, organization and toxicity of the possible prefibrillar aggregation states is given. In section 3., the hypothesis of the possible role in $\mathrm{AD}$ of the $\mathrm{N}$-truncated and pyromodified $\mathrm{A} \beta$ is described in comparison to full-length $A \beta$. In section 4., the inhibition of the most toxic prefibrillar aggregation states is discussed as a new therapeutic strategy in AD. In section 5., concluding remarks are reported on the possible new molecular therapeutic strategy in $\mathrm{AD}$, based on the inhibition of the most toxic soluble $\mathrm{A} \beta$ oligomeric aggregation states.

\section{Oligomeric aggregation states of beta amyloids in Alzheimer's disease}

In this section we review recent advancements in the description and characterization of soluble oligomeric aggregates, their toxicity and possible role in Alzheimer pathogenesis (Campioni et al., 2010; Ono et al., 2009; Mastrangelo et al., 2006; Roychaudhuri et al., 2009; Sandbergh et al., 2010).

Soluble oligomers, as fractionated by size exclusion chromatography, are often classified as low molecular weight oligomers (<10 monomers) and high molecular weight oligomers (in the range of 20-40 monomers) (Mastrangelo et al., 2006; Jahn et al., 2010; Walsh et al., 1999). Efforts to establish rigorous structure-toxicity correlations have been hindered by the complex, dynamic equilibria displayed by A $\beta$ (Ono et al., 2009; Roychaudhuri et al., 2009) . To clarify this matter it is therefore preliminarly necessary to choose selected well-defined, stabilized oligomers for establishing rigorous structure activity correlations leading to knowledge-based therapeutic drug design (Ono et al., 2009). The technique of photoinduced cross-linking of unmodified proteins (PICUP) was used to stabilize oligomers of A $\beta 1-40$ (Ono et al., 2009). The results are summarized quantitatively in Table1 where the oligomeric structure-toxicity characterization for uncross-linked, cross-linked, small oligomers and fibrils is reported from reference (Ono et al., 2009).

The data suggest that monomer/dimer transition involves the largest change in the conformation content, from random to $\beta$-sheet conformation, as observed by circular 
dichroism (CD); while the three dimensional change in the dimensions (TEM and AFM) of the oligomers has its maximum in the dimer/trimer and trimer/tetramer transitions.

\begin{tabular}{|c|c|c|c|c|c|c|}
\hline Sample & $\alpha$-Helix ${ }^{\star}$ & $\beta$-Sheet* & $S C^{*}$ & Diametert & Height* & $E C 50^{5}$ \\
\hline Uncross-linked & 9.4 & 25.2 & 65.4 & $1.15 \pm 0.17(79)$ & $0.14 \pm 0.01(135)$ & $102.5 \pm 5.6$ \\
\hline Cross-linked & 11.1 & 33.6 & 55.3 & $12.39 \pm 2.13(55)$ & $2.93 \pm 0.40(56)$ & $43.0 \pm 2.7$ \\
\hline Pure monomer & 8.7 & 24.0 & 67.3 & $1.30 \pm 0.13(127)$ & $0.24 \pm 0.01(178)$ & $67.3 \pm 8.7$ \\
\hline Pure dimer & 10.5 & 38.6 & 50.9 & $1.78 \pm 0.23(116)$ & $0.53 \pm 0.03(165)$ & $41.6 \pm 3.9$ \\
\hline Pure trimer & 10.3 & 40.8 & 48.9 & $7.22 \pm 1.12(64)$ & $0.94 \pm 0.13(67)$ & $24.5 \pm 1.9$ \\
\hline Pure tetramer & 12.7 & 44.9 & 42.4 & $11.00 \pm 2.08(39)$ & $1.51 \pm 0.30(38)$ & $20.5 \pm 0.4$ \\
\hline Fibrils & ND & 57.0 & 43.0 & NO & ND & $57.6 \pm 2.2$ \\
\hline
\end{tabular}

ND, not determined.

^CD data are expressed as percent of each secondary structure element.

tMean diameter $\pm S E$, in $n m$, is listed for $(n) A B$ assemblies visualized by $E M$.

tMean height $\pm S E$, in $n m$, is listed for $(n) A \beta$ assemblies visualized by AFM.

SEffective concentration ( $E C_{50}$ ) is the concentration of a particular $A \beta$ assembly that produced a level of toxicity in $M T T$ assays that was half maximal. ECSD [mean concentration $(\mu \mathrm{M}) \pm S E$ ] values were calculated after sigmoidal curve fitting of the data shown in Fig. 55 , using GraphPad Prism software (version 4.0a).

\section{Table 1. Characteristics of $A \beta$ assemblies}

Finally MTT analysis (last column in Table 1, represented as the concentration in $\mu \mathrm{M}$ of a particular assembly that produced a level of toxicity in MTT assays that was half maximal) says that both cross-linked oligomeric $A \beta$ and fibrillar $A \beta$ were significantly more toxic than unassembled $A \beta$ (dimers three-fold and tetramers 13-fold more toxic than monomers). One of the main results is the confirmation that monomers have very low toxicity activity while only when $A \beta$ self associate, toxicity rises substantially, even though it is impossible to establish a degree of increasing toxicity with the number of monomers in the given oligomer because of the decrease in occurrence frequency of higher order oligomers. The authors suggest as a possible therapeutic strategy in $\mathrm{AD}$ to target the low order oligomers to unstabilize the most toxic $A \beta$ contributing to AD neurotoxicity (Ono et al., 2009).

Monomeric $\mathrm{A} \beta$ has the propensity to adopt in water solutions different conformations, including transient extended $\beta$-sheet conformations in the central and C-terminal regions, connected by turn between them, or $\beta$-hairpin (see Figure 1, panel A) (Hou et al., 2004; Mitternachet et al., 2010; Sandbergh et al., 2010).

When a synthetic or purified and denatured monomeric $A \beta$ (say the full length peptide $A \beta 1$ 42 or the C-terminal truncated $A \beta 1-40$ or the N-terminal truncated $A \beta 3-42$ and modified A $\beta p y 3-42, \ldots)$ is incubated in a native buffer, peptide aggregates are formed showing oligomeric species increasing in molecular weight, initially found by $\mathrm{CD}$ in a disordered (random) conformation that may transiently convert into $\beta$-sheet oligomers that assemble further into protofibrils, following distinct pathways according to the original monomer peptide. Some of these transient oligomeric aggregation states may be built by subunits in the hairpin conformation after conversion of intramolecular Hbonds and antiparallel $\beta$-strands into intermolecular Hbonds and parallel $\beta$-sheets (Hoyer et al., 2008). By engineering a double cysteine mutant, in which the $\beta$-hairpin is stabilized by an intramolecular disulfide bond, $\mathrm{A} \beta 1$ 40 and $A \beta 1-42$ are transformed into $A \beta 40 C C$ and $A \beta 42 C C$, becoming building block of toxic $A \beta$ oligomers, unable to convert into amyloid fibrils (Sandbergh et al., 2010) (see figure 1).

In figure 1 , panel $A$, the transient $\beta$-hairpin is first compared with the stabilized $\beta$-hairpin by the two disulfide bonds in the double cystein mutants, in panel B (ThT fluorescence assay) it is shown that $A \beta 40 C C$ is unable to transform, after incubation, in fibrils being blocked in the 
oligomeric form (see relative TEM micrograpghs, panel C), evolution that is recovered after treatment of $A \beta 40 C C$ with the reducing agent TCEP. The neurotoxicity of different $A \beta C C$ aggregates was assayed by measuring their ability to induce apoptosis (level of caspase-3/7 activity in human neuroblastoma cell line, SH-SY5Y) (Sandbergh et al., 2010). A $\beta 42 C C$ aggregates, pooled from SEC, all induce apoptosis $24 \mathrm{hr}$ after addition to the cells, but $\beta$-sheet oligomer fractions are considerably more toxic than low molecular weight or high molecular weight fractions of $A \beta 42 C C$ : the $\beta$-sheet oligomers $($ at $1 \mu \mathrm{M})$ resulted 50 times more potent than wild-type $A \beta 42$ monomer or fibril samples (see figure 2).

A

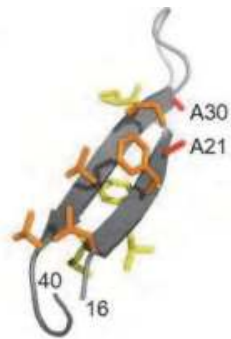

Wild type $A \beta$

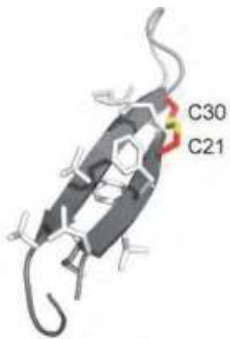

$\mathrm{A} \beta \mathrm{CC}$

B
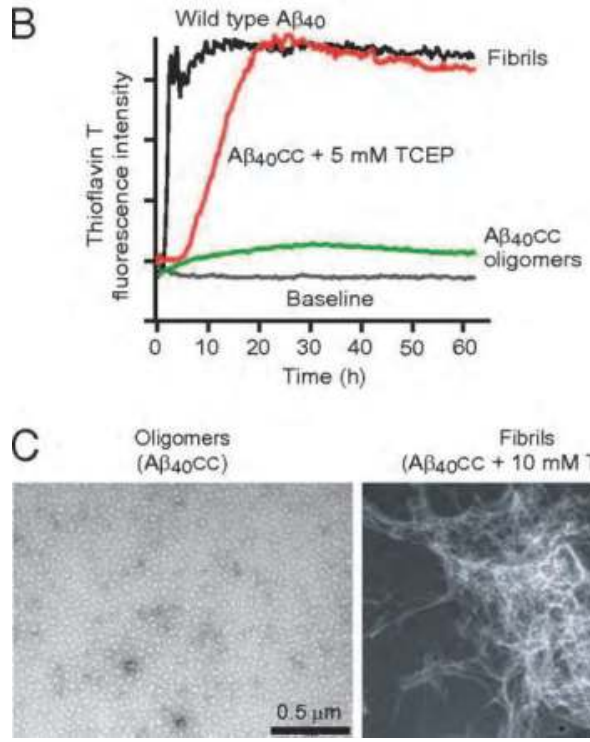

Fibrils $(\mathrm{A} \beta 40 \mathrm{CC}+10 \mathrm{mM}$ TCEP)

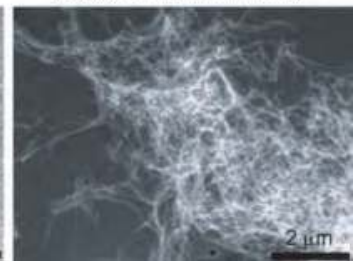

Fig. 1. Panel A. On the left, the $\beta$-hairpin conformation of $A \beta_{40}$ in complex with an Affibody binding protein [29]; the ALA21 and ALA30 methyls are located in close proximity on opposite $\beta$-strands. On the right, model of double-mutated A $\beta$ A21C and A30C, A $\beta C C$, in which the $\beta$-hairpin conformation is locked by a disulfide bond. Panel $B$. ThT fluorescence assays of $A \beta_{40} C C$ aggregation in the absence or presence of reducing agent (TCEP), compared with wild-type $A \beta_{40}$ aggregation. Panel C. TEM micrographs of $\beta$-sheet oligomers of $A \beta_{40} C C$ (left) and of fibrils formed in presence of TCEP (right). Adapted from Figure 1 of reference (Sandbergh et al., 2010). 

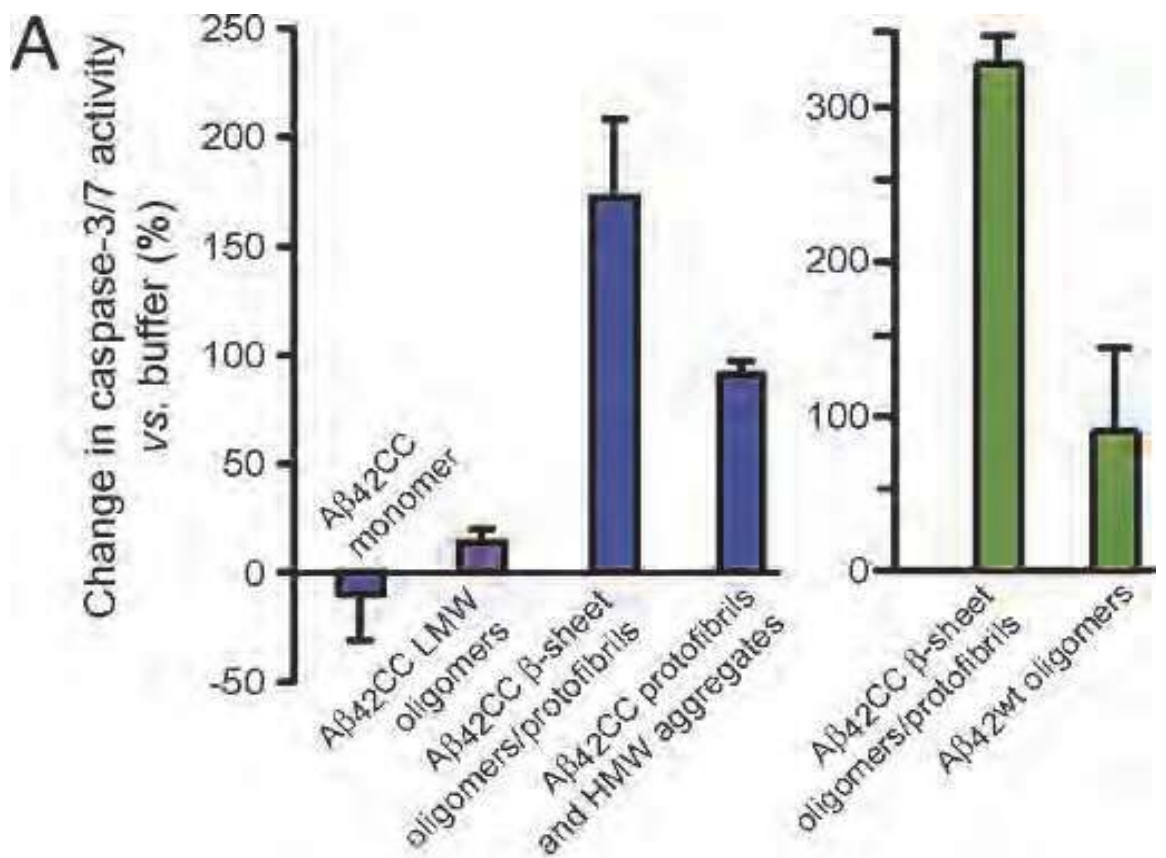

Fig. 2. Neutoxicity of A $\beta C C$ to SH-SY5Y human neuroblastoma cells. Caspase-3/7 activity reporting on apoptosis was measured after $24 \mathrm{~h}$ of treatment with $10 \mu \mathrm{M}$ of different $\mathrm{A} \beta_{42} \mathrm{CC}$ species (blue); $\mathrm{A} \beta_{42} \mathrm{CC}$ oligomers compared with wild-type $\mathrm{A} \beta_{42}$ (green). Adapted from Figure 4 of reference (Sandbergh et al., 2010).

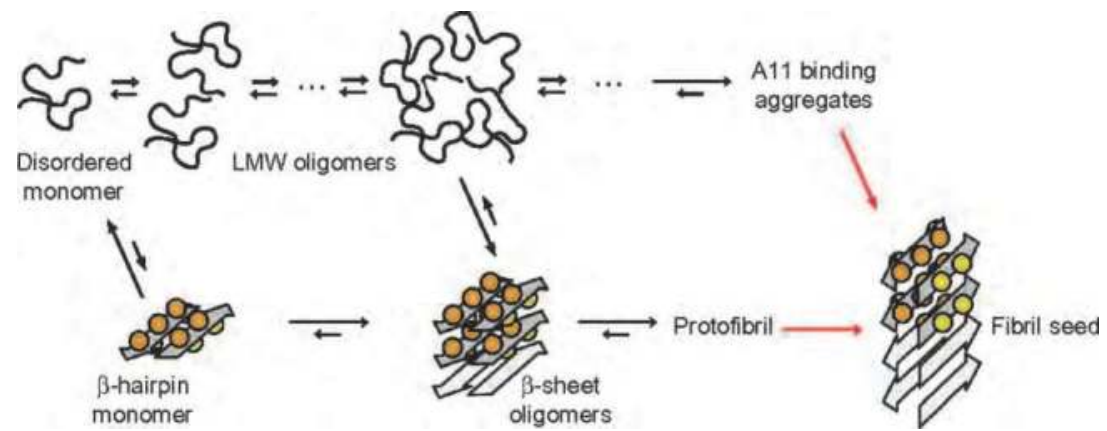

Fig. 3. A $\beta$ aggregation via two pathways. Disordered pathway: upper; toxic $\beta$-sheet oligomers pathway: lower. Adapted from Figure 5 of reference (Sandbergh et al., 2010).

In conclusion an aggregation scheme was proposed as synthesized in figure 3 (Sandbergh et al., 2010). It is possible that the aggregation and kinetic plasticity of $A \beta$ leads to different pathways for amyloid formation, having different impacts on AD pathogenesis (figure 3): one pathway involves disorganized oligomers and large non fibrillar aggregates (disordered less toxic pathway) and the other involves assembly in $\beta$-sheet oligomers which in turn may 
be the building blocks of protofibrils as the penultimate intermediate before fibril seeds formation (toxic $\beta$-sheet oligomers pathway). A $\beta 42 \mathrm{CC}$ more readily forms $\beta$-sheet oligomers than $A \beta 40 C C$, probably as a result of the presence of a kinetic barrier to oligomer formation that can only be overcome by A $340 C C$ with the aid of heating and concentration (Sandbergh et al., 2010). It is possible that the stronger toxicity found for $A \beta 42$ in respect to $A \beta 40$ reflects different barriers to $\beta$-sheet oligomer formation. Accordingly, the former peptide may follow the toxic $\beta$-sheet oligomers pathway while the latter may follow the disordered pathway. This concept of kinetic partitioning of $A \beta$ into two or more aggregation pathways may be important in AD progress, and at the same time the choice between the different pathways is very subtle because morphologically very similar oligomers may show different cell toxicity. A demonstration of this hyperfine behaviour was finely given by considering a protein model, the 91 residue N-terminal domain HypF-N of Escherichia coli (Campioni et al., 20 10).

This protein, prepared in different conditions, gives two stable oligomers HypF-N-A and HypF-N-B of similar morphological and tintorial properties but with the first one very toxic and the second one almost non toxic versus human neuroblastoma cells (SH-SY5Y), via MTT and Hend assays (figure 4, panel A) (Campioni et al., 2010).

To gain insight into the structural differences of the two oligomers HypF-N-A and HypF-N-B, 18 mutational variants of the protein were expressed, each carrying a single cysteine residue but located at different positions along the polypeptide chain, and labeled with a pyrene. Each labeled variant was allowed to aggregate separately, analysing the resulting fluorescence spectra with the eventual formation of pyrene excimers as a probe of the proximity (about 1 $\mathrm{nm}$ ) of labelled pairs. It was shown that the structural hydrophobic core of the non-toxic HypF-N-B oligomers is more compact and less solved exposed (see figure 4, panel B) than the less tightly packed core of the toxic HypF-N-A oligomers. This demonstrates that the difference in cell membrane interaction and ability to cause cell dysfunction is due to the small differences in structural flexibility and solvent exposure of the core residues.

Toxicity is associated with the ability of the oligomeric species to form a more pronounced and disruptive interaction with cells, by exposure to the solvent of the surfaces of hydrophobic disorganized residues and thus leading to cell death. Thus, the protein model HypF-N has allowed oligomeric states, that would normally be metastable, to be trapped and therefore studied, with conclusions probably valid for the toxic properties of many oligomers in disease-related systems (Campioni et al., 2010).

Summarizing (Campioni et al., 2010; Sandbergh et al., 2010), to form amyloid-like structures is generic to polypeptide chains, but whether or not these species are pathogenic depends on their structural features, notably the extent to which hydrophobic residues are flexible and exposed on their surfaces within the environment of a living organism. Thus it seems that for therapeutic purposes the toxicity can be substantially reduced if the hydrophobic residues are incorporated to a greater extent within the interior of the oligomeric assemblies, even in the absence of an effective change in morphology: solvent-exposed structurally disorganized hydrophobic residues within small protein oligomers are at the origin of the pathogenesis of important human diseases.

Nevertheless the world of $A \beta$ assembly still is too complex to be conclusive on the related biological activity in neurodegenerative diseases and fundamental questions remains to be clarified such as: is $A \beta$ the main proximate etiologic agent of $A D$ ?; what is the structure of the proximate neurotoxic $A \beta$ assembly? (Roychaudhuri et al., 2009). 

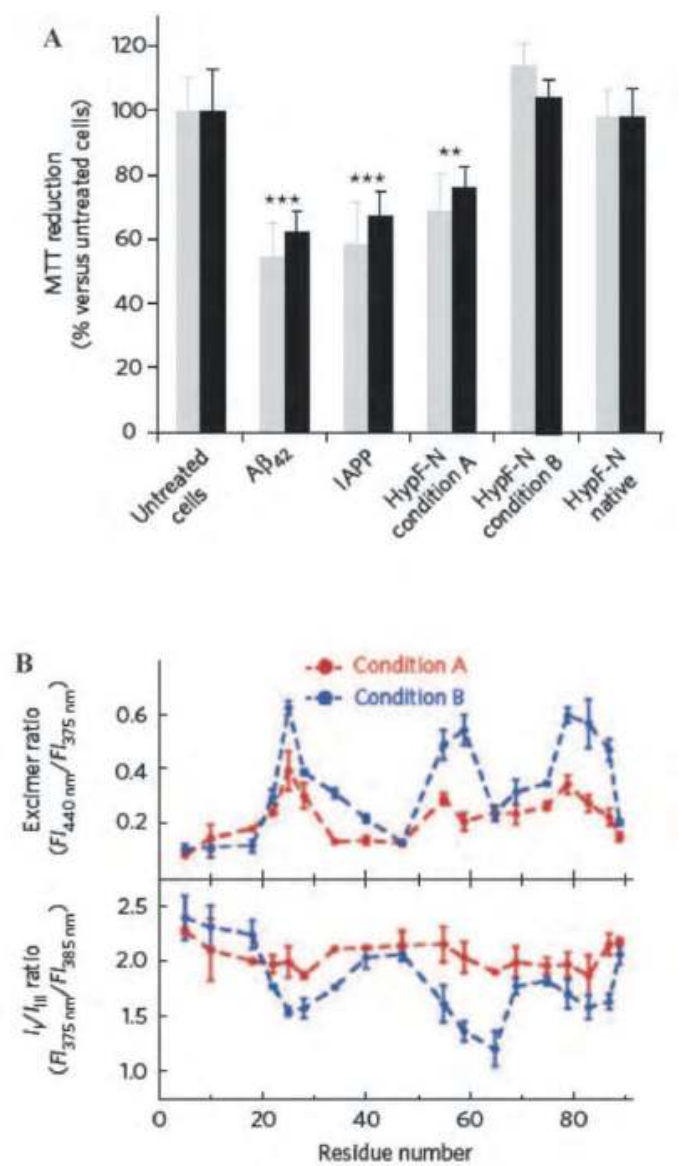

Fig. 4. Toxicity of HypF-N aggregates. Panel A. MTT reduction assay on SH-SY5Y (gray) and Hend (black) cells untreated (lane 1), or treated with A $\beta 1-42$ oligomers (lane 2), control IAPP oligomers (lane 3), HypF-N aggregates under condition A (lane 4) and B (lane 5) and native HypF-N (lane 6). Panel B. Pyrene fluorescence emission spectra: excimer ratio profiles of HypF-N oligomers formed under condition A (red) and B(blue). Adapted from Figures 1 and 3 of reference (Campioni et al., 2010).

One of the main problems is to well characterize the structure of oligomeric states even when well followed: normally this is done by TEM and AFM with a lack of resolution to characterize such small and dynamic structures. Recently, a new approach in AFM was developed at LifeAFM , Port Jefferson (NY), that yields high-resolution images and allows to observe the structure of the smallest MW oligomers (Mastrangelo et al., 2006). The new AFM methodology, at difference with conventional tapping mode AFM, is based on a single low-force touch of the cantilever tip, enabling a non-destructive imaging of $A \beta$ and observation of the small Mw oligomers. AFM of A $\beta 42$ at early incubation time, give images of monomers, dimers, trimers, tetramers and other low Mw oligomers that are consistent with the hairpin structure, above described (Figure 5) (Mastrangelo et al., 2006). 

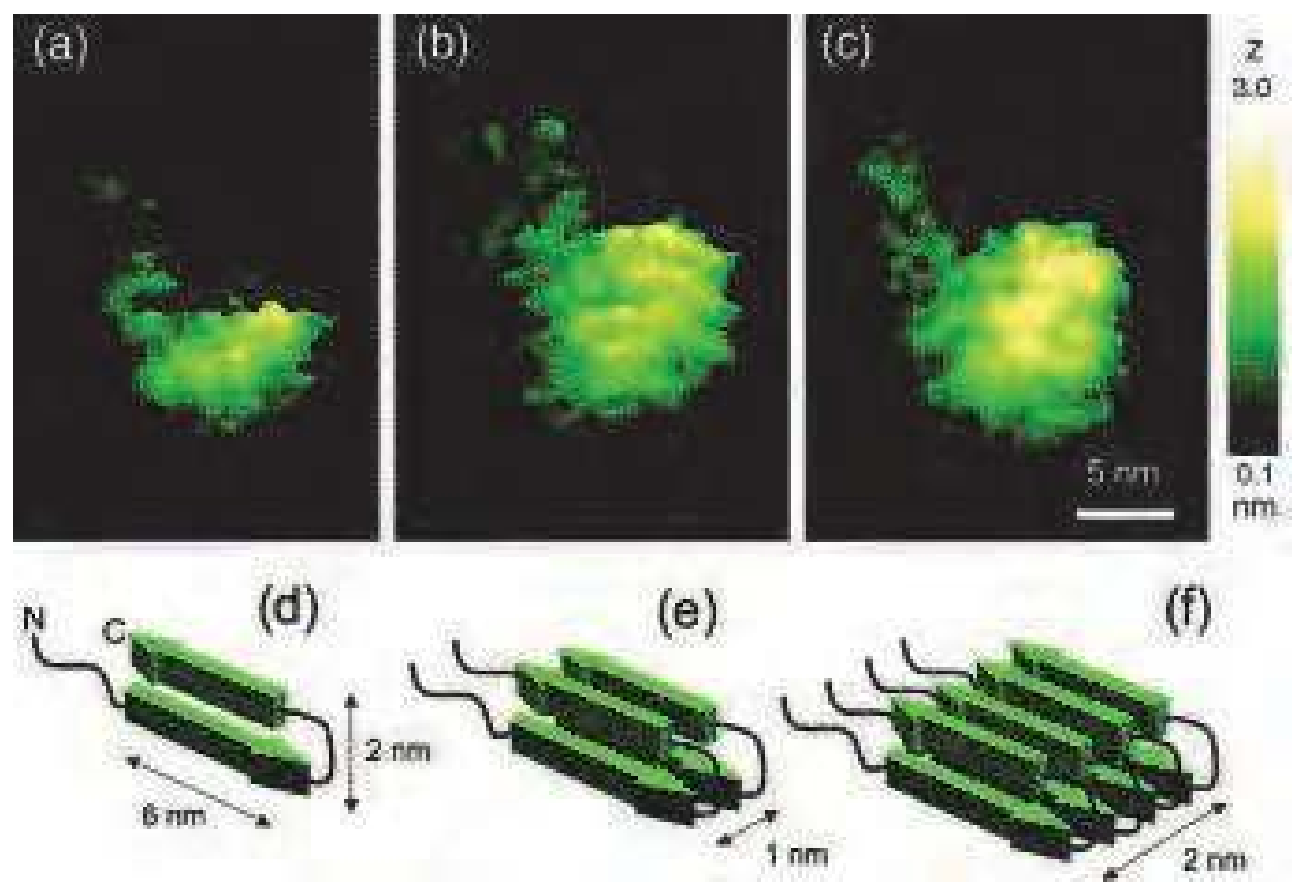

Fig. 5. High resolution AFM of A $\beta 1-42$. AFM images are interpreted as panel A: a dimer; panel $B$ and C: a tetramer. In each image the diffuse area to the left of the particle is interpreted as the disordered N-terminal region of the A $\beta 1-42$ monomer. Adapted from Figure 4 of reference (Mastrangelo et al., 2006).

For the first time, images of such small oligomers were obtained, that may be interpreted as hairpin monomer, dimer and tetramer (see panels $d$, e and f). Note that the structures described above were found also in Alzheimer brains and should be present in the toxicity studies to clarify what structural features of the soluble oligomers are responsible of toxicity (Mastrangelo et al., 2006).

\section{Comparison of $\mathrm{N}$-truncated and pyromodified $A \beta$ to full-length $A \beta$ aggregation states: Transient aggregation states and kinetics}

The N-terminal truncated and pyroglutamyl modified $A \beta$, as summarized in the introduction, accounts for the majority of the deposited peptides and of the most toxic effects on neurons. Recently, it was demonstrated that, in similar initial buffer conditions and using three different techniques (flow cytometry, ThT fluorescence and circular dichroism), these N-terminal modified peptides aggregate much faster than the fulllength $A \beta$ (D'Arrigo et al., 2009; Shilling et al., 2006). Altogether, the aggregation kinetics of A $\beta p y 3-42$ was found 20-250 by cytometry and ThT fluorescence (Shilling et al., 2006) and more than 30 times by CD (D'Arrigo et al., 2009) faster than that of A $\beta 1-42$ respectively, depending upon the assay method. The oligomers that form $\beta$-structure rapidly are found to be more toxic (D'Arrigo et al., 2009; Mastrangelo et al., 2006; Shilling et al., 2006). 

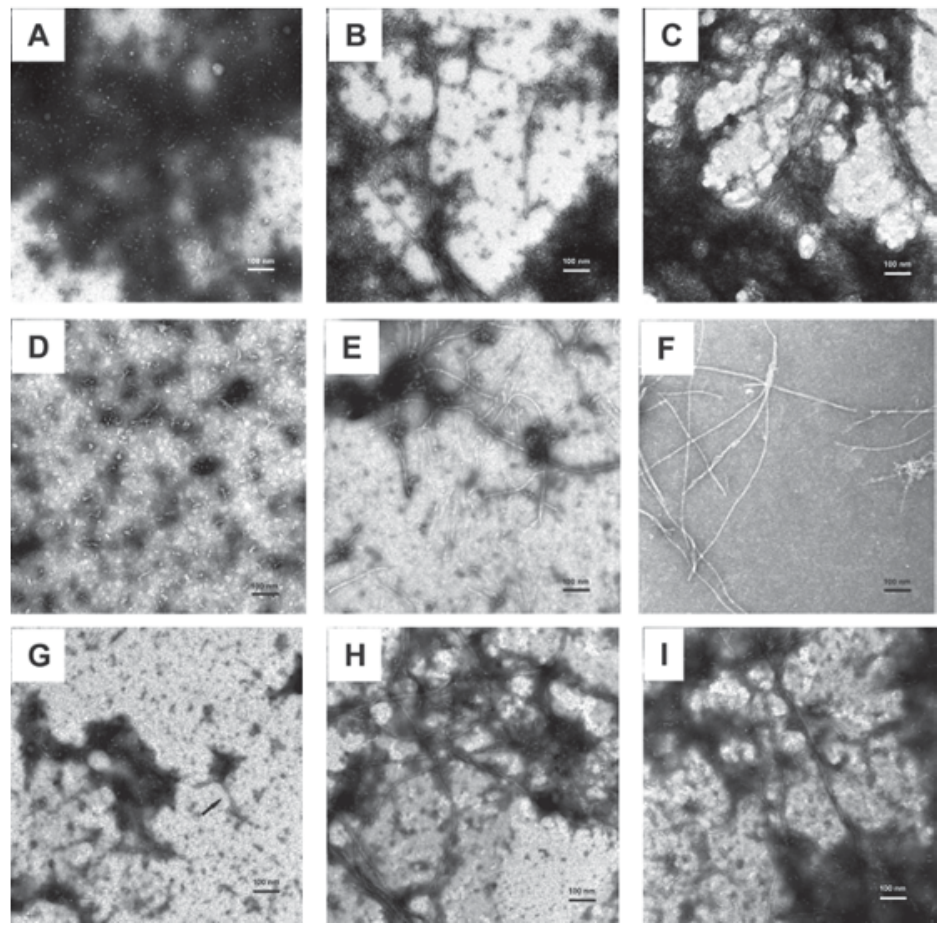

Fig. 6. The peptide TEM morphology after incubation in $20 \mathrm{mM}$ phosphate buffer, $\mathrm{pH} 7.4$ at $37^{\circ} \mathrm{C}$. A $31-42$ : panel A, at initial incubation time; panel B, after 1 day of incubation; panel C, after 2 days of incubation. A $\beta p y 3-42$ : panel D, at initial incubation time; panel $\mathrm{E}$, after 1 day of incubation; panel $F$, after 2 days of incubation. The 1:1 mixture A $\beta 1-42 / A \beta p y 3-42$ : panel $\mathrm{G}$, at initial incubation time (the arrow points to a protofibril); panel $\mathrm{H}$, after 1 day incubation; panel I, after 2 days of incubation. Peptides concentrations: $50 \mu \mathrm{M}$. Adapted from Figures 6 and 7 of reference (D'Arrigo et al., 2009).

In addition the aggregation pathway of the two $A \beta$ leads to more toxic dynamic oligomeric states for $A \beta p y 3-42$ and to fibril formation for $A \beta 1-42$, see for instance (D'Arrigo et al., 2009) and (He \& Barrow, 1999), while when the two are mixed (e.g. in a 1:1 ratio) A $\beta p y 3-42$ has an inhibitory effect on the fibrillogenesis of A $31-42$, favouring prefibrillar aggregation states, that are more toxic for the cells (see Figure 6) (D'Arrigo et al., 2009).

These experiments confirm the strong role of A $\beta p y 3-42$ on the progression of Alzheimer pathogenesis ( $\mathrm{D}^{\prime}$ Arrigo et al., 2009; Jan et al., 2008).

The $A \beta$ peptides display regions that are more affected by environmental conditions which may be responsible for conformational transitions and for different aggregation states and kinetics towards fibrillization (D'Arrigo et al., 2009). This has been confirmed experimentally, especially by NMR (Danielsson et al., 2006; Hou et al., 2004; Tomaselli et al., 2006), and it can be well illustrated by a simple model using the basic properties of the 20 aminoacids. In Figure 7 a model of the two $A \beta$ peptides, $A \beta 1-42$ and $A \beta p y 3-42$, is reported ( $D^{\prime}$ Arrigo et al., 2009), as a string of pearls where each pearl represents one amino acid. Each aminoacid has one of 5 colours, each colour corresponding to the 5 generic standard classification of the 20 aminoacids: red, negatively charged residue; blue, positively charged residue; yellow, polar 
residue; black, hydrophobic residue; white, Gly residue. The C-terminals of the three peptides and the N-terminal of $A \beta 1-42$ are represented by red and blue squares respectively.

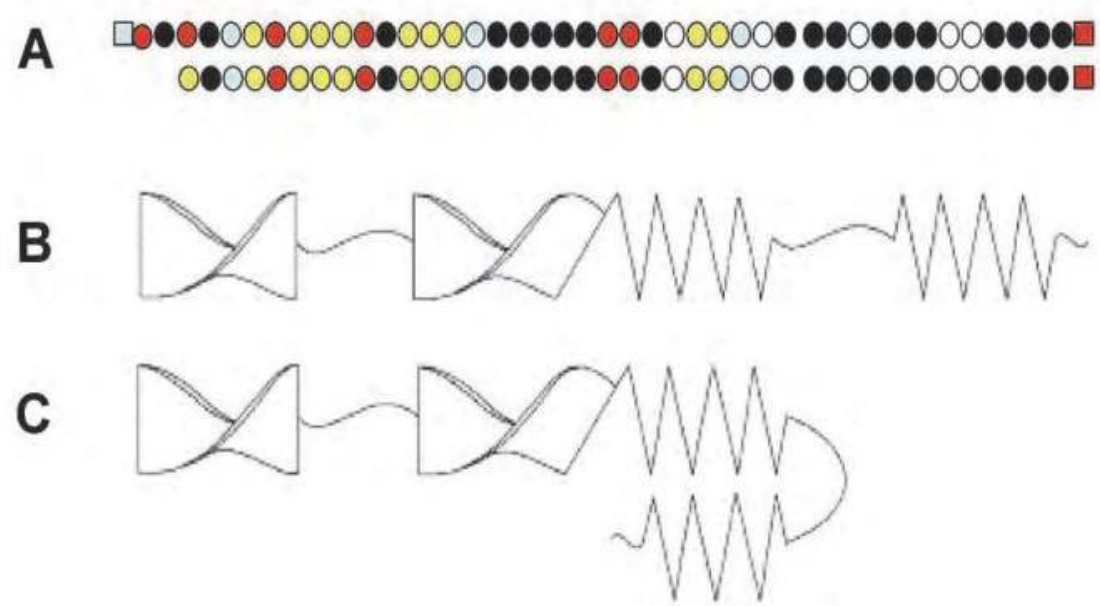

Fig. 7. Five colors representation pearl chain model of A $\beta 1-42$ and A $\beta p y 3-428$ (panel A) . Red pearl: negatively charged residue; Blue pearl: positively charged residue; Yellow pearl: polar residue; Black pearl: hydrophobic residue; White pearl: Gly residue. The Cterminals of the two peptides and the N-terminal of $A \beta 1-42$ are represented by red and blue squares respectively. Conformation of $A \beta 1-40$, according to NMR analysis (panel B) of ref. [32]. The proposed $\beta$-hairpin structure of toxic oligomers (panel C), see ref. [32]. Adapted from Figure 9 of reference (D'Arrigo et al., 2009) and Figure 6 of reference (Danielsson et al., 2006).

The central hydrophobic region 16-24 is crucial for the $\beta$-sheet forming oligomerization and subsequent fibril formation (Danielsson et al., 2006) and is well identified in the 5-colour representation. On the contrary the N-terminal region 1-16 has a large propensity, demonstrated by NMR, to be in an extended (especially at low temperature, where, accordingly, $A \beta$ is found more soluble) PII-helix which is retained going from apolar to polar environments, or binding metal ions, or partially retained raising the temperature (Danielsson et al., 2006; Tomaselli et al., 2006). Again the NMR findings have a rough correspondence with the 1-16 sequence in the 5 colour representation of A $\beta 1-42$, even though the correlation is less strong. Reducing or removing the helix propensity of the Nterminal region may favor a faster and different aggregation. One way to realize this enhancement of the aggregation process is just to destabilize the N-terminal region of $A \beta 1$ 42 by truncating the first two residues and by pyroglutamyl termination to A $\beta p y 3-42$. Going from $A \beta 1-42$ to $A \beta p y 3-42$ the $N$-terminal portion of $A \beta$ suffers important and indicative changes: the negative charges decrease from 7 to 5 , the positive charges from 4 to 3 , globally the terminal chain loosing 3 charges, while the hydrophobic residues decrease only by one in favour of an equal increase in polar residues. On this basis one may infer (D'Arrigo et al., 2009) that the helix propensity of the N-terminal is strongly reduced and the peptide, in which the central hydrophobic part did not make any change, is now more prone to the $\beta$ conformational state and to aggregation, as observed by CD (D'Arrigo et al., 2009; He \& Barrow, 1999). 
Different $A \beta$ peptides, full-length and truncated and modified, do have different attitude to develop the cross- $\beta$ structure conformation with big consequences not only on fibrillization but also on the aggregation kinetics and on the formation of different prefibrillar aggregation states. So these N-terminal truncations and pyroglutamil modifications of A $\beta 1-42$ have the consequences of accelerating the initial aggregation kinetics, directing it towards faster oligomerization. In addition, the py-Glu residue prevents degradation of the peptide by aminopeptidase (Shilling et al., 2004, 2006) and at the same time, as demonstrated by TEM, A $\beta p y 3-42$ inhibits the great propensity of the full-length peptide to aggregate in fibrils, favouring and stabilizing pre-fibrillar aggregation states that may be very toxic for the cells and for the progression of Alzheimer pathogenesis (D'Arrigo et al., 2009).

\section{Inhibition of the most toxic prefibrillar aggregates as a new therapeutic strategy against Alzheimer pathogenesis}

From sections 2 and 3 it comes out that a possible new therapeutic strategy against AD may be based on the inhibition of the highest toxic prefibrillar aggregates (Ono et al., 2009). As we have seen before, this means that attention should be directed to reduce the $A \beta$ prefibrillar aggregations particularly of those more stable components which are organized in such a way to be greatly toxic to neuron cells. This logic suggests to target ordered oligomers and small ordered aggregates, destabilizing them in favour of disorder states. This is something that applies to all the forms of $A \beta$ peptides, but it should be even of more importance to target special peptides such as the N-terminal truncated and pyroglutamyl modified peptides like A $\beta p y 3-42$. This strategy was strongly pursued by Demuth's group. In a remarkable 2008 paper on Nature Medicine (Shilling et al., 2008), Demuth and coll. Indicate, by experiments in vivo and in vitro, that inhibition of the level of glutaminyl cyclase (QC) in mammalian brains, where it is widely distributed, attenuates pyroglutamate $\mathrm{A} \beta$ and AD pathology.

To assess whether QC expression can be correlated with generation of A $\beta p y 3-42$ in AD, QC mRNA and protein concentrations in human neocortical brain samples post mortem were analysed (figure 8A). It was found that QC mRNA and protein were upregulated in samples from AD individuals, compared with samples from normal aging individuals; significantly larger concentrations of $A \beta p y 3-42$ were detected by ELISA analysis in the former individuals relative to the latter (figure $8 \mathrm{~B}$ ). This supports the role of $\mathrm{QC}$ in the generation of $\mathrm{A} \beta p y 3-42$. In contrast high total $A \beta x-42$ concentrations in aged controls were found (figure $8 \mathrm{~B}$ ).

The protein APP and QC were co-expressed in HEK293 cells to study the correlations between QC expression and A $\beta p y 3-42$ generation (Shilling et al., 2008). When the first potent inhibitor for human QC, PDB150 (Buchholz et al., 2006), was added, the A $\beta p y 3-42$ formation was suppressed the most the highest the inhibitor dose, while the other non pyromodified peptides were not affected (figure 8C). This demonstrates the specific effect of the inhibitor on QC catalysis (figure 8C and 8D).

Subsequently PBD150 was orally applied to 4-month-old female Tg2576 mice for 6 months to study effects of QC inhibition on the A $\beta p y 3-42$ and on non pyromodified peptides in the insoluble $A \beta$ pool (Shilling et al., 2008). A $\beta$ py3 -42 decreased by $23 \%$ and $65 \%$ according to the PBD150 dose as well as the non pyromodified peptides. Two are therefore the indications in vivo: $\mathrm{A} \beta p y 3-42$ generation is mediated by $\mathrm{QC}$ and there is a correlation between $A \beta p y 3-42$ aggregation and total $A \beta$ aggregation.

These interesting conlusions were confirmed by other experiments on the effects of QC inhibition on Tg256 mice and on TASD-41 mice (Shilling et al., 2008). 
It seems to us that the conclusions of the authors of the above paper (Shilling et al., 2008), that prevention of pyroglutamate $\mathrm{A} \beta$ at the $\mathrm{N}$-terminus by $\mathrm{QC}$ inhibition represents a new therapeutic strategy for alleviating amyloidoses caused by the seeding of amyloidogenic peptides, was well substantiated. This may represent an important step to get a first molecular strategy in AD therapy.
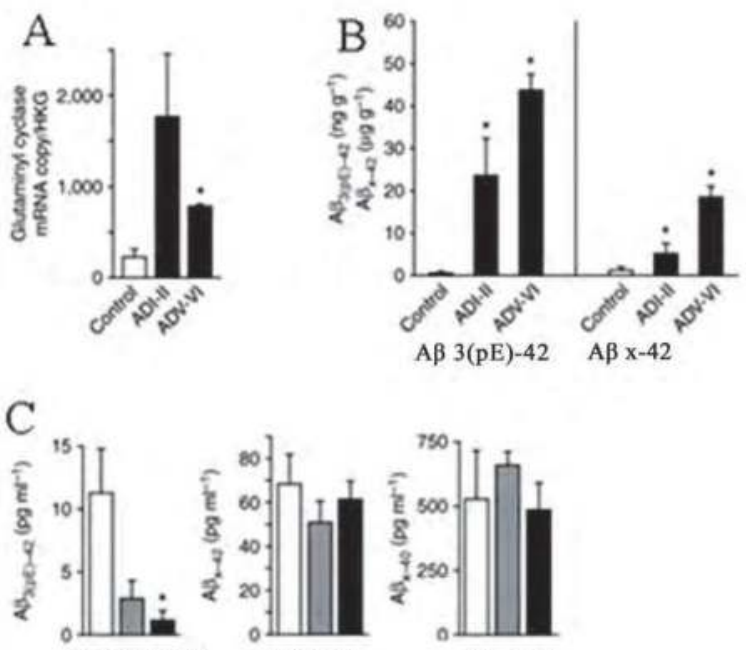

$\mathrm{A \beta} 3(\mathrm{pE})-42$
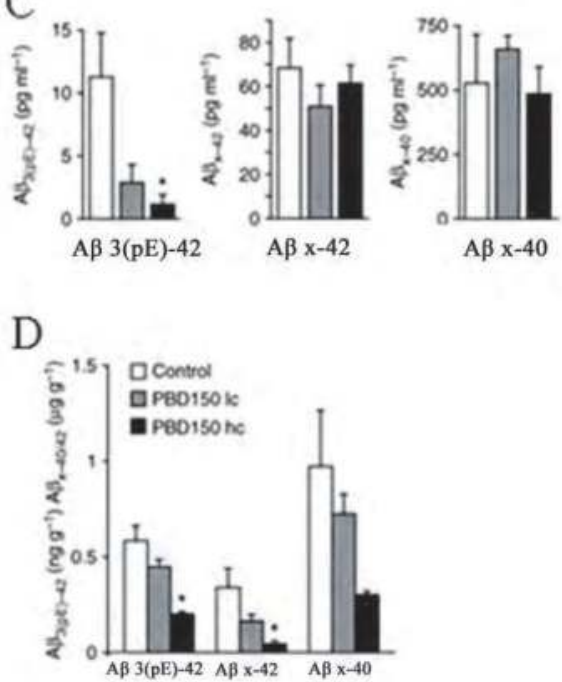

Fig. 8. Glutamynil cyclase (QC) expression and pyroglutamate-modified N-terminally truncated $A \beta$ : prevention of $A \beta p y$ formation by glutamynil cyclase inhibition in vitro and in vivo. Panel A: quantitative analysis of QC transcript levels. Total RNA from human neocortical brain samples was isolated from aged controls and individuals with AD (the Roman numerals in ADI, ADII, ADV-VI refer to Braak stages). Panel B: Quantification of A $\beta p y 3-42$ and A $\beta 1-42$ concentrations from the samples of Panel A by ELISA analysis. Panel $C$ : quantification of $A \beta$ concentrations in conditioned media of HEK293 cells, which were transiently transfected with an APPsw / 1 variant and human QC. PBD150 lc (low concentration $)=0.1 \mu \mathrm{M}$; (high concentration $)=1 \mu \mathrm{M}$. Panel D: quantification of $\mathrm{A} \beta$ concentrations in the brains of 10-month old Tg2576 control mice and of age-matched littermates treated for 6 months with PBD150 at a concentration of $2.4 \mathrm{mg}$ per $\mathrm{g}$ (PBD150lc) or $7.2 \mathrm{mg}$ per $\mathrm{g}$ (PBD150lc) of food pellets. Only female mice were enrolled in the study. Adapted from Figure 1 of (Shilling et al., 2008). 


\section{Conclusion}

We firstly introduced the prefibrillar aggregation states that are thought to be responsible of the progress of Alzheimer's pathogenesis, and then discussed the possible peptide conformation and assembly organization of them. These features has been correlated with their peptide content, particularly full-length A $\beta 1-42$ and N-truncated and modified A $\beta p y 3-42$, their structure, kinetics and cell toxicity. We have reviewed the elements of a new therapeutic strategy generically based on opposing conformational diseases taking into account properly of the conformational and structural organization of most toxic oligomeric aggregates. One of the most toxic oligomeric aggregates is formed by A $\beta p y 3-$ 42: the new therapeutic strategy may be the prevention of pyroglutamyl termination by finding a well tolerated inhibitor of glutamynil cyclase, which is responsible of the catalysis in vivo of this termination. More difficult is to find conformational inhibitors of the more extended variety of amyloid peptides participating to the formation of the most toxic oligomeric aggregation states. If some steps in the new therapeutic strategy have been done, more work is required to determine more extensively the conformation and structure of the possible oligomeric states and their interaction with neuronal cells to clarify the mechanism of toxicity.

Finally, we have not treated here this topic, but evidence exists that metals are involved as well, in the pathogenesis of AD but the mechanism and role, although important it remains unclear.

\section{Acknowledgement}

Finantial support from Compagnia di San Paolo Foundation (Torino, Italy) in the framework of BASKO project is acknowledged.

\section{References}

Apetri, M. M.; Maiti, N.C.; Zagorski, M.G.; Carey, P.R; Anderson, V.E. (2006) Secondary structure of alpha-synuclein oligomers. Characterization by raman atomic force microscopy. J. Mol. Biol. 355, 63-71.

Buchholz, M., Heiser, U., Shilling S., Niestroj, A. J., Zunkel, K., Demuth, H. U. (2006). The first potent inhibitors for human glutaminyl cyclase: synthesis and structureactivity relationship J. Med. Chem. 49, 664-677.

Campioni, S., Mannini, B., Zampagni, M., Pensalfini, A., Parrini, C., Evangelisti, E., Relini, A., Stefani, M., Dobson C. M., Cecchi, C., Chiti, F. (2010). A causative link between the structure of aberrant protein oligomers and their toxicity. Nature Chem. Biol. 6, 140-147.

Chiti, F.; Taddei, N.; Baroni, F.; Capanni, C.; Stefani, M.; Ramponi, G.; Dobson, C. M. (2002).Kinetic partitioning of protein folding and aggregation. Nature Structural Biology 9, 137-143.

Chiti F., Dobson C. M. (2006). Protein misfolding, functional amyloid, and human disease. Annu. Rev. Biochem. 75, 333-366. 
Danielsson, J.; Andersson, A.; Jarvet, J.; Graslund, A. (2006). ${ }^{15} \mathrm{~N}$ relaxation study of theamyloid beta-peptide: struictural propensities and persistence length. Magn. Reson. Chem. 44, S114-S121.

D'Arrigo C., Tabaton, M., Perico, A. (2009). N-terminal truncated pyroglutamyl beta amyloid peptide Abetapy3-42 shows a faster aggregation kinetics than the fulllength Abeta1-42 Biopolymers 91, 861-873.

Forloni, G.; Terreni, L.; Bertani, I.; Fogliarino, S.; Invernizzi, R.; Assini, A.; Ribizzi, G.; Negro, A.; Calabrese, E.; Volontè, M.A.; Mariani, C.; Franceschi, M.; Tabaton, M.; Bertoli, A. (2002). Protein misfolding in Alzheimer's and Parkinson's disease: genetics and molecular mechanisms. Neurobiol. Aging, 23, 957-976.

He, W.; Barrow, C. J. (1999). The Abeta 3-pyroglutamyl and 11-pyroglutamyl peptides foundin senile plaque have greater beta-sheet forming and aggregation propensities in vitro than full-length Abeta. Biochemistry 38, 10871-10877.

Hou L., Shao, H., Zhang, Y., Li, H., Menon, N. K., Neuhaus, E., B., Brewer, J. M., Byeon, I. L., Ray, D. G., Vitek, M. P., Iwashita, T., Makula, R. A., Przybyla, A. B., Zagorski, M. G. (2004). Solution NMR studies of the Abeta(1-40) and Abeta(1-42) peptides establish that the Met35 oxidation state affects the mechanism of amyloid formation. J. Am. Chem. Soc. 126, 1992-2005.

Hoyer V., Gronwall C., Jonsson A., Stahl S., Hard T. (2008). Stabilization of a beta-hairpin in monomeric Alzheimer's amyloid-beta peptide inhibits amyloid formation. Proc. Natl. Acad. Sci. USA 105, 5099-5104.

Jahn, A., Hartley, D. M., Lashuel, H. A. (2010). Preparation and characterization of toxic Abeta aggregates for structural and functional studies in Alzheimer's disease research. Nature protocols 5, 1186-1209.

Jan A., Gokce O., Luthi-Carter R., Lashuel H. A. (2008). The ratio of monomeric to aggregated forms of Abeta40 and Abeta42 is an important determinant of amyloidbeta aggregation, fibrillogenesis, and toxicity. J. Biol. Chem. 283, 28176-28189.

Kirkitadze M.D., Bitan G., Teplow D.B. (2002). Paradigm shifts in Alzheimer's disease and other neurodegenerative disorders: the emerging role of oligomeric assemblies. $J$. Neurosci. Res. 69, 567-577.

Mastrangelo, I. A., Ahmed, M., Sato, T., Liu, W., Wang, C., Hough, P., Smith, S. O. (2006). High-resolution atomic force microscopy of soluble Abeta42 oligomers. J. Mol. Biol. 358, 106-119.

Mitternacht S., Staneva I., Hard T., Irback A. (2010). Comparing the folding free-energy landscapes of Abeta42 variants wityh different aggregation properties. Proteins 78, 2600-2608.

Ono, K. Condron, M.M., Teplow, D. B. (2009). Structure-neurotoxicity relationships of amyloid beta-protein oligomers. PNAS 106, 14745-14750.

Pastor, M. T., Kummerer, N., Schubert, V., Esteras-Chopo, A., Dotti, FC. G., Lopez de la Paz, M., Serrano, L. (2008). Amyloid toxicity is independent of polypeptide sequence, length and chirality. J. Mol. Biol. 375, 695-707.

Roychaudhuri, R. Yang, M., Hoshi, M. M., Teplow, D. B. (2009). Amyloid beta-protein assembly and Alzheimer disease. J. Biol. Chem. 284, 4749-4753. 
Russo, C.; Schettini, G.; Saido, T. C.; Hulette, C.; Lippa, C.; Lannfelt, L.; Ghetti, B.; Gambetti,P.; Tabaton, M.; Teller, J.K. (2000). Presenilin-1 mutations in Alzheimer's disease. Nature 405, 531-532.

Russo, C.; Violani, E.; Salis, F.; Venezia, V.; Dolcini, V.; Damonte, G.; Benatti, U.; D’Arrigo, C.; Patrone, E.; Carlo, P.; Schettini, G. (2002). Pyroglutamate-modified amyloid beta-peptides-AbetaN3(pE)-strongly affect cultured neuron and astrocyte survival. J. Neurochem. 82, 1480-1489.

Sandbergh, A., Luheshi, L. M., Sollvander, S., Pereira de Barros, T., Macao, B., Knowles, T. P. J., Biverstal, H., Lendel, C., Ekholm-Petterson, F., Dubnovitsky, A., Lannfelt, L., Dobson, C. M., Hard, T. (2010). Stabilization of neurotoxic Alzheimer amyloid-beta oligomers by protein engineering. PNAS 107, 1559515600.

Selkoe D. J. (1996). Amyloid beta-protein and the genetics of Alzheimer's disease. J.Biol. Chem. 271, 18295-18298.

Selkoe D. J. (2004). Alzheimer disease: mechanistic understanding predicts novel therapies. Ann. Intern. Med. 140, 627-638.

Shankar G. M., Metha T. H., Garcia-Munoz, A., Shepardson, N. E., Smith, I., Brett, F. M., Farell, M. A., Rowan, M. J., Lemere, C. A., Regan, C. M., Walsh, D. M., Sabatini, B. L., Selkoe, D. J. (2008). Amyloid-beta protein dimmers isolated directly from Alzheimer's gbrains impair synaptic plasticity and memory. Nat. Med. 14, 837842.

Schilling, S.; Hoffmann, T.; Manhart, S.; Hoffmann, M.; Demuth, H.U. (2004). Glutamynil cyclase unfold glutamyl cyclase activity under mild acid conditions. FEBS Lett. 563, 191-196.

Schilling, S., Lauber, T., Schaupp, M., manhart, S., Scheel, E., Bohm, G., Demuth H. U. (2006). On the seeding and oligomerization of pGlu-amyloid peptides. Biochemistry 45, 12393-12399.

Shilling S., Zeitschel, U., Hoffmann, T., Heiser, U., Francke, M., Kehlen, A., Holzer, M., Hutter-Paier, B., Prokesh, M., Windisch, M., Jagla, W., Schlenzig, D., Lindner, C., Rudolph, T., Reuter, G., Cynis, H., Montag, D., Demuth, H. U., Rossner, S. (2008). Glutaminyl cyclase inhibition attenuates pyroglutamate Abeta and Alzheimer's disease-like pathology. Nature Medicine, Letters 14, 1106.

Tomaselli, S.; Esposito, V.; Vangone, P.; van Nuland, N. A. J.; Bonvin, A.M.; Guerrini, R.; Tancredi, T.; Temussi, P.A.; Picone, D. (2006). The alfa-to-beta conformational transition of Alzheimer's Abeta(1-42) peptide in acqueous media is reversible: a step by step conformational analysis suggests the location of beta conformation seeding. ChemBioChem 7, 257-267.

Walsh, D. M., Hartley, D. M., Kusumoto, Y., Fezoui, Y., Condron M. M., Lomakin, A., Benedek, G. B., Selkoe, D. J., Teplow, D. B. (1999). Amyloid beta-protein fibrillogenesis. Structure and biological activity of protofibrillar intermediates. J. Biol. Chem. 274, 25945-25952.

Walsh D. M., Selkoe D.B. (2007). A beta oligomers-a decade of discovery. J. Neurochem. 101,1172-1184. 
Yankner B. A., Lu T. (2009). Amyloid beta-protein toxicity and the pathogenesis of Alzheimer disease. J. Biol. Chem. 284, 4755-4759.

Yoshiike, Y., Akagi, T., Takashima, A. (2007). Surface structure of amyloid-beta fibrils contributes to cytotoxicity. Biochemistry 46, 9805-9812. 


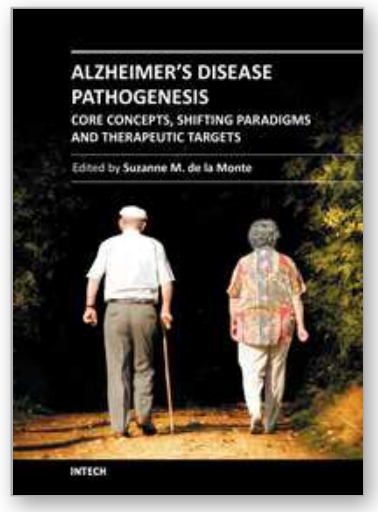

\author{
Alzheimer's Disease Pathogenesis-Core Concepts, Shifting \\ Paradigms and Therapeutic Targets \\ Edited by Dr. Suzanne De La Monte
}

ISBN 978-953-307-690-4

Hard cover, 686 pages

Publisher InTech

Published online 12, September, 2011

Published in print edition September, 2011

Alzheimer's Disease Pathogenesis: Core Concepts, Shifting Paradigms, and Therapeutic Targets, delivers the concepts embodied within its title. This exciting book presents the full array of theories about the causes of Alzheimer's, including fresh concepts that have gained ground among both professionals and the lay public. Acknowledged experts provide highly informative yet critical reviews of the factors that most likely contribute to Alzheimer's, including genetics, metabolic deficiencies, oxidative stress, and possibly environmental exposures. Evidence that Alzheimer's resembles a brain form of diabetes is discussed from different perspectives, ranging from disease mechanisms to therapeutics. This book is further energized by discussions of how neurotransmitter deficits, neuro-inflammation, and oxidative stress impair neuronal plasticity and contribute to Alzheimer's neurodegeneration. The diversity of topics presented in just the right depth will interest clinicians and researchers alike. This book inspires confidence that effective treatments could be developed based upon the expanding list of potential therapeutic targets.

\title{
How to reference
}

In order to correctly reference this scholarly work, feel free to copy and paste the following:

Angelo Perico, Denise Galante and Cristina D'Arrigo (2011). Structure and Toxicity of the Prefibrillar Aggregation States of Beta Peptides in Alzheimer's Disease, Alzheimer's Disease Pathogenesis-Core Concepts, Shifting Paradigms and Therapeutic Targets, Dr. Suzanne De La Monte (Ed.), ISBN: 978-953-307690-4, InTech, Available from: http://www.intechopen.com/books/alzheimer-s-disease-pathogenesis-coreconcepts-shifting-paradigms-and-therapeutic-targets/structure-and-toxicity-of-the-prefibrillar-aggregationstates-of-beta-peptides-in-alzheimer-s-diseas

\section{INTECH}

open science | open minds

\section{InTech Europe}

University Campus STeP Ri

Slavka Krautzeka 83/A

51000 Rijeka, Croatia

Phone: +385 (51) 770447

Fax: +385 (51) 686166

www.intechopen.com

\section{InTech China}

Unit 405, Office Block, Hotel Equatorial Shanghai

No.65, Yan An Road (West), Shanghai, 200040, China 中国上海市延安西路65号上海国际贵都大饭店办公楼 405 单元

Phone: +86-21-62489820

Fax: $+86-21-62489821$ 
(C) 2011 The Author(s). Licensee IntechOpen. This chapter is distributed under the terms of the Creative Commons Attribution-NonCommercialShareAlike-3.0 License, which permits use, distribution and reproduction for non-commercial purposes, provided the original is properly cited and derivative works building on this content are distributed under the same license. 